\title{
Failure is Also an Option
}

\author{
Antoine Amarilli ${ }^{1}$, Marc Beunardeau ${ }^{2}$, \\ Rémi Géraud $^{2}$, and David Naccache ${ }^{2}$ \\ 1 Institut Mines-Télécom; Télécom ParisTech; CNRS LTCI \\ 46 rue Barrault, F-75634 Paris CEDEx 13, France \\ given_name.family_name@telecom-paristech.fr \\ 2 École normale supérieure \\ Département d'informatique \\ 45 rue d'Ulm, F-75230 Paris CEDEx 05, France \\ given_name.family_name@ens.fr
}

\begin{abstract}
The Nijmeegse Vierdaagse ${ }^{1}$ is the world's most famous walking event. The walk is known to be challenging and each year about $10 \%$ of the participants drop out. In 2016 the Vierdaagse will celebrate its centennial anniversary. In the walker community there is a frenzy about participating in the centennial walk. Initially, the rules governing participation were the following: A walker who succeeds the $n$-th walk is admitted to walk at year $(n+1)$. Walkers who fail a walk enter a lottery. If they win the lottery, they are also granted tickets to the walk. Finally, walkers who fail two successive draws are admitted to the walk following the second lottery failure. In 2013, while computing our chances to be admitted to the centennial walk, we noticed a rather counterintuitive fact: By purposely failing the 97 -th walk, walkers can actually... increase their chances to attend the centennial walk.
\end{abstract}

We notified this inconsistency to the organizers. We never got an answer but the rules were subsequently changed.

\section{Introduction}

The Nijmeegse Vierdaagse is the world's most famous walking event. The walk is known to be challenging and each year about $10 \%$ of the participants drop out. In 2016 the Vierdaagse will celebrate its centennial anniversary.

In the walker community there is a frenzy about participating in the centennial walk. Initially, the rules governing participation were the following:

Let $\mathcal{P}$ be a participant.

\footnotetext{
${ }^{1}$ http://www . 4daagse.nl/en/
} 
- Rule 1: If $\mathcal{P}$ finishes the walk of year $n$ then $\mathcal{P}$ is admitted to the walk on year $n+1$.

- Rule 2: If $\mathcal{P}$ fails the walk of year $n$ then $\mathcal{P}$ enters a lottery:

- If $\mathcal{P}$ wins the lottery then $\mathcal{P}$ is admitted to walk of year $n+1$.

- If $\mathcal{P}$ fails the lottery then $\mathcal{P}$ is excluded from the walk of year $n+1$.

- Rule 3: If $\mathcal{P}$ fails the two successive lotteries of years $n$ and $n+1$ then $\mathcal{P}$ is admitted to walk at year $n+2$.

Rule 3 is meant to avoid a situation where, by sheer bad luck, a person will never be given a chance to attend the Vierdaagse.

On the eve of the 97-th walk we wished to estimate our odds to reach the centennial walk. While doing so, we noticed a rather counter-intuitive fact: By purposely failing the 97-th walk, walkers can actually... increase their chances to attend the centennial walk.

Interestingly, this strategy does not depend on one's walking skills nor on the chances to succeed the lottery.

\section{Failing, to Succeed...}

\subsection{The underlying Markov chain}

The rules define a Markov chain having two terminal states: being admitted to the centennial walk, and not being admitted. Let $W, W, L, L$ denote the following events:

- $W: \mathcal{P}$ walked and succeeded

$-W: \mathcal{P}$ walked and failed

- $L: \mathcal{P}$ participated in lottery and won

$-L: \mathcal{P}$ participated in lottery and failed

We distinguish two categories of scenarios: those leading to the centennial walk, and those that do not. Let the probability to succeed a walk be $w=\operatorname{Pr}[W]$ and let the probability to be selected during a draw be $\ell=\operatorname{Pr}[L]$. Naturally $\operatorname{Pr}[W]=1-w$ and $\operatorname{Pr}[L]=1-\ell$.

The transition graph is described in Table 1 and Figure 1.

\subsection{The Optimal Strategy}

If we sum the probabilities of the events leading to the centennial walk we get that the probability $A$ to arrive to the centennial walk is:

$$
\begin{aligned}
A=1 & -2 \ell+3 \ell^{2}-\ell^{3}-w+4 \ell w-4 \ell^{2} w+\ell^{3} w+\ell w^{2}-2 \ell^{2} w^{2} \\
& +\ell^{3} w^{2}+w^{3}-3 \ell w^{3}+3 \ell^{2} w^{3}-\ell^{3} w^{3}
\end{aligned}
$$




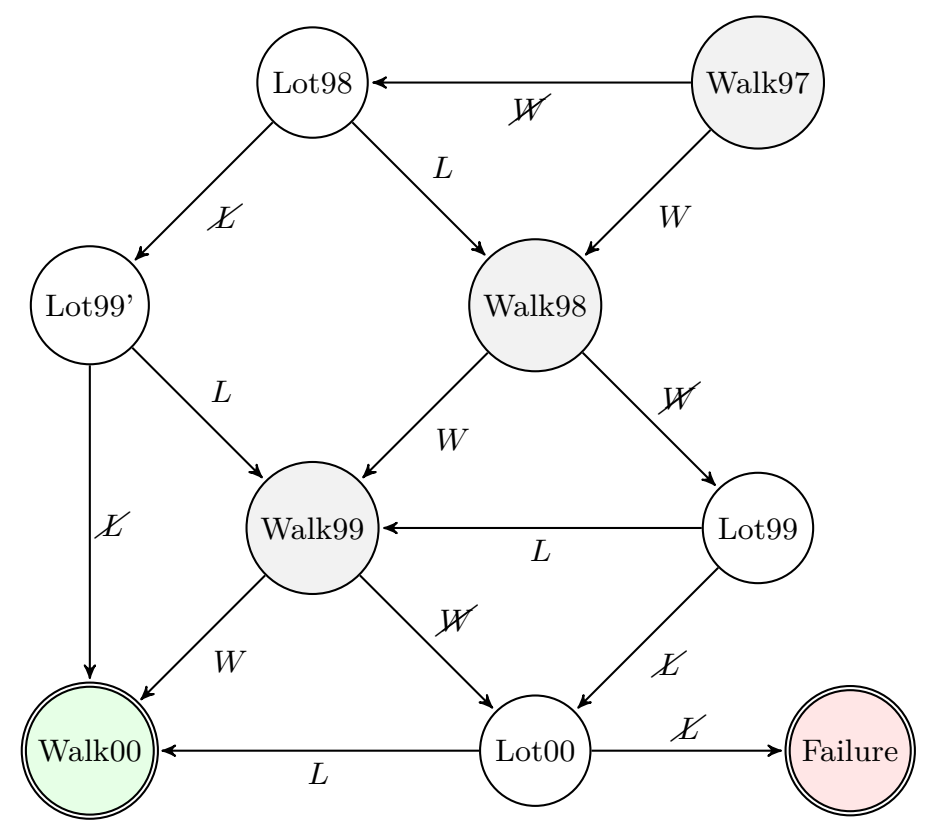

Fig. 1. Transition graph for the centennial walk, starting from the 1997 walk.

The honest reader would usually stop reading here. A cryptographer seeking for algorithmic loopholes would notice that there is a further option: Purposely fail the 97-th walk!

What would happen then? The Markov chain is simplified as in Table 2 and Figure 2.

If we sum the probability of events leading to the centennial walk we get that the new probability to arrive to the centennial walk is:

$$
B=1-2 \ell+3 \ell^{2}-\ell^{3}+\ell w-\ell^{2} w+\ell w^{2}-2 \ell^{2} w^{2}+\ell^{3} w^{2} .
$$

Let us determine the values of $\ell, w$ for which $B>A$. It turns out that:

$$
B>A \Rightarrow(\ell-1)^{3} w\left(w^{2}-1\right)>0
$$

Since $w \geq 0$ is a positive probability this is equivalent to:

$$
(\ell-1)^{3}\left(w^{2}-1\right)>0 \Rightarrow(\ell-1)\left(w^{2}-1\right)>0
$$

And because $\ell$ and $w$ are probabilities (which are smaller than 1), this inequality is... always satisfied!

Hence, to optimize the chances to get the centennial ticket, the best thing to do is... purposely fail the 97 -th walk! and this independently of one's walking skills (i.e., it is valid for all $w$ )! 
Table 1. Transition graph to the centennial walk.

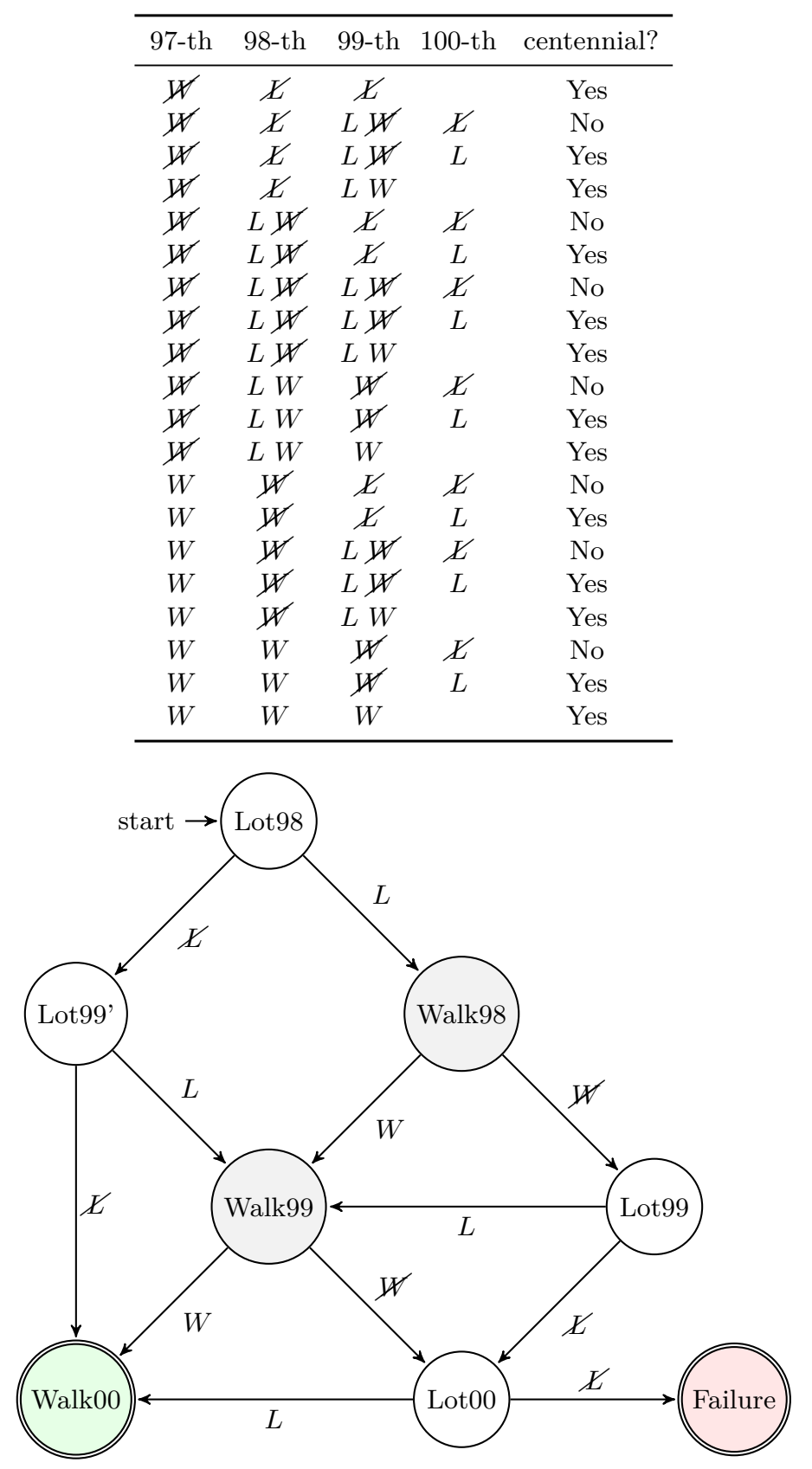

Fig. 2. Transition graph for the centennial walk, starting from the 1998 lottery. 
Table 2. Simplified transition graph after failing the 97 th walk.

\begin{tabular}{|c|c|c|c|}
\hline 98-th & 99-th & 100-th & centennial? \\
\hline L & L & & Yes \\
\hline L & $L W$ & L & No \\
\hline L & $L W$ & $L$ & Yes \\
\hline L & $L W$ & & Yes \\
\hline$L W$ & L & $E$ & No \\
\hline$L \not H$ & K & $L$ & Yes \\
\hline$L W$ & $L \not$ & L & No \\
\hline$L W$ & $L W$ & $L$ & Yes \\
\hline$L W$ & $L W$ & & Yes \\
\hline$L W$ & W & X & No \\
\hline$L W$ & W & $L$ & Yes \\
\hline$L W$ & $W$ & & Yes \\
\hline
\end{tabular}

\section{Aftermath}

We notified this inconsistency to the organizers. We never got an answer but the rules were subsequently changed. As we write these $\operatorname{lines}^{2}$ :

“... Walkers who have successfully completed the Four Days Marches at least four times between 2010 and 2015 will be guaranteed a starting ticket for the 100-th edition ..."

The main problem is Rule 3 . This rule, meant to protect walkers from a strike of bad luck, opens a loophole to the centennial walk. Despite the modifications made to the rules, this loophole still exists: The most successful path, for a weak walker, is through the lotteries. Indeed, lotteries provide a guaranteed win at some probability which is completely independent from the walker's abilities.

Note that even if walking skills and lottery probabilities vary in time, it still pays to fail on purpose. Denoting by $\ell_{i}$ the probability to win the lottery at year $i$ and by $\omega_{i}$ the probability to finish the walk of year $i$. We get an updated inequality for $B>A$ :

$$
\left(\ell_{100}-1\right)\left(\ell_{98}-1\right)\left(\ell_{99}-1\right) \omega_{97}\left(\omega_{98} \omega_{99}-1\right)>0
$$

Which is, again, always true for all $\ell_{i}, \omega_{i}$ values. This also implies that failing on purpose is useful even when we take into account the option of purposedly failing later walks, which amounts to setting $\omega_{i}=0$ for some values of $i>97$.

\footnotetext{
${ }^{2}$ http: //www . 4daagse.nl/en/home.html?id=273
} 
Is there a simple way to both reward strong walkers while not penalizing bad luck? We make the following suggestion: Let the lottery probability $p_{i}$ for a walker $\mathcal{P}$ depend on their number of successful walks $k$, and the number of years since their last selection $a$, in a way that grows lexicographically:

$$
(k, a) \mapsto 1-\frac{1}{k+1}+\frac{1}{(k+1)(k+2)}\left(1-\frac{1}{a+1}\right)
$$

Of course, the probability must be adjusted depending on the desired number of participants to select to a walk; the principle is just that the probability of a walker to succeed the lottery should depend lexicographically on $k$ and $a$. This should intuitively guarantee that losing on purpose is never a better idea than walking, because walking gives a better chance of winning a later lottery.

This solution might not be perfect, however. For one thing, good walkers have better chances to be selected in a lottery; as a quick fix, instead of $k$ we could use $N-k$, i.e. count the number of failed walks. Such a function acts as an incentive not to deliberately fail: The outcomes are better if one finishes the walk rather than relying on the lottery. 